\title{
Hot Jupiter secondary eclipses measured by Kepler
}

\author{
B.-O. Demory ${ }^{1} \&$ S. Seager ${ }^{1}$ \\ ${ }^{1}$ Department of Earth, Atmospheric and Planetary Sciences, Massachusetts \\ Institute of Technology, Cambridge, USA [demory@mit.edu]
}

\begin{abstract}
Hot-Jupiters are known to be dark in visible bandpasses, mainly because of the alkali metal absorption features. The outstanding quality of the Kepler mission photometry allows a detection (or non-detection upper limits on) giant planet secondary eclipses at visible wavelengths. We present such measurements on published planets from Kepler Q1 data. We then explore how to disentangle between the planetary thermal emission and the reflected light components that can both contribute to the detected signal in the Kepler bandpass. We finally investigate how different physical processes can lead to a wide variety of hot-Jupiters albedos.
\end{abstract}

\section{Background and motivation}

Secondary eclipses of two dozen transiting hot-Jupiters have been observed at infrared wavelengths with the Spitzer Space Telescope so far (see, e.g., Seager \& Deming 2010). These strongly irradiated planets efficiently absorb visible light from their host stars and exhibit temperatures that largely exceed 1000K. The hot Jupiters consequently produce infrared emission signature of the order of $10^{-3}$ as compared to the host star flux.

The reflected light component of the hot-Jupiter population is critical to constrain planetary energy budgets and to explore the upper atmosphere properties. While Jupiter has a geometric albedo of 0.5 in the visible, HD209458b's geometric albedo is surprisingly low : $<0.08$ (3- $\sigma$ upper limit, Rowe et al. 2008). Establishing a survey of secondary eclipses in the visible is thus highly desirable to better understand the origins of such diversity.

Table 1 summarizes visible band geometric albedo measurements that have been obtained to date. Hot Jupiters are dark, due to the alkali metal ( $\mathrm{Na}$ and $\mathrm{K}$ ) line absorption and possibly due to $\mathrm{TiO}$ and $\mathrm{VO}$ strong molecular absorption bands in the visible (e.g. Seager \& Sasselov 2000, Marley et al. 1999, Sudarsky et al. 2000, 2003).

The planetary to stellar flux ratio of hot Jupiters at visible wavelengths is of the order of $10^{-5}$, making it very challenging to measure (see Fig.1). After just 1.5 years of operation, Kepler has proven to be a facility able to achieve a few parts per million (ppm) photometric precision (e.g. Jenkins et al., 2010). We present an overview of secondary eclipse measurements for published Kepler giant planets and explore the possible origins of the signature measured in visible wavelengths.

This is an Open Access article distributed under the terms of the Creative Commons Attribution-Noncommercial License 3.0, which permits unrestricted use, distribution, and reproduction in any noncommercial medium, provided the original work is properly cited. 


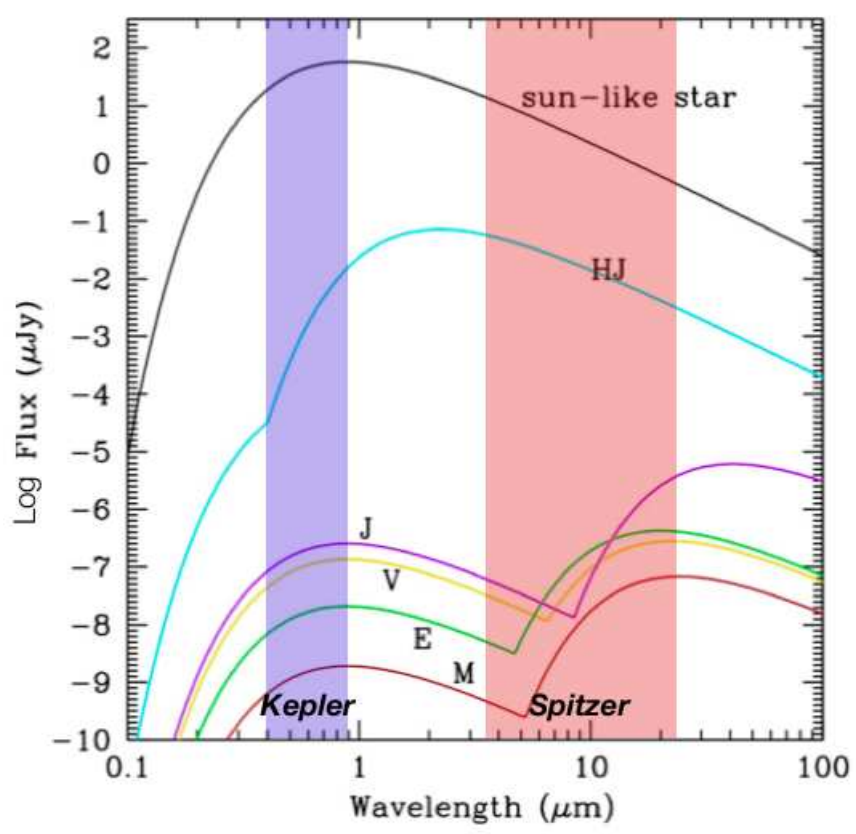

Figure 1: Approximate spectral energy distribution for the Sun, Jupiter, Venus, Mars, the Earth and a representative hot Jupiter. Both thermal and reflected components are plotted for each planet. Bandpasses of Kepler and Spitzer are indicated, showing which wavelength region of the planetary spectra are probed. Adapted from Seager 2003.

\section{Geometric albedo determination}

We performed Markov Chain Monte-Carlo analyses on the 6 hot Jupiters that have been observed by Kepler, including two previously known exoplanets : TrES-2b and HATP-7b. Public data from the first quarter were used to derive the systems parameters. Geometric albedos were computed from the secondary eclipse depth, planetary radius, and semi-major axis (eq. 1, Lopez-Morales \& Seager 2007). The geometric albedo is wavelength-dependent and measures the ratio of the planet flux at zero phase angle to the flux from a Lambert sphere at the same distance and the same cross-sectional area as the planet (see Seager 2010).

$$
\frac{F_{p}}{F_{\star}}=A_{g}\left(\frac{R_{p}}{a}\right)^{2}
$$

The photometric precision of Kepler is illustrated in Fig. 2, where a single, $80 \mathrm{ppm}$ secondary eclipse of HAT-P-7b is shown and clearly detected. Results from this preliminary study appear in Table 2 and depict a wide variety of geometric albedos, ranging from 0.06 to 0.35 . Such analysis has been performed by Kipping \&Bakos (2010) and results show good consistency with those presented here.

Before any interpretation regarding scattered light contribution, one should cautiously examine to which extent the measured depth encompasses a thermal component. 
Table 1: Published constraints on hot Jupiters geometric albedos measured in the visible.

\begin{tabular}{llcr}
\hline Planet & Geometric Albedo & $\begin{array}{c}T_{e q} \\
{[\mathrm{~K}]}\end{array}$ & Reference \\
\hline$\tau$ Bootis b & $0.32 \pm 0.13\left(R_{P}=1.2 R_{J u p}\right)$ & $\sim 1500 \mathrm{~K}$ & Leigh et al. 2003a \\
$"$ & $<0.3\left(R_{P}=1.2 R_{\text {Jup }}\right)$ & $"$ & Charbonneau et al. 1999 \\
HD75289 & $<0.12(3-\sigma$ upper limit $)$ & 1260 & Leigh et al. 2003b \\
HD209458b & $<0.08(3-\sigma$ upper limit $)$ & 1550 & Rowe et al. 2008 \\
CoRoT-1b & $<0.20(3-\sigma$ upper limit $)$ & 2330 & Snellen et al. 2010 \\
CoRoT-2 & $0.06 \pm 0.06$ & 1910 & Alonso et al. 2010 \\
\hline
\end{tabular}

Table 2: Geometric albedos and equilibrium temperatures (assuming no redistribution) for Kepler published giant planets from public data (Q1).

\begin{tabular}{llc}
\hline Planet & Geometric Albedo & $\begin{array}{l}T_{e q} \\
{[\mathrm{~K}]}\end{array}$ \\
\hline Kepler 5b & $0.21 \pm 0.10$ & 1557 \\
Kepler 6b & $0.18 \pm 0.09$ & 1411 \\
Kepler 7b & $0.35 \pm 0.11$ & 1370 \\
Kepler 8b & $0.21 \pm 0.10$ & 1567 \\
TrES-2b & $0.06 \pm 0.05$ & 1464 \\
HAT-P-7b & $0.20 \pm 0.03$ & 2085 \\
\hline
\end{tabular}

\section{Disentangling thermal emission and reflected light}

As illustrated in Fig. 3 with the case of Kepler-5b, hot-Jupiter thermal emission could have a significant contribution to the planetary flux measured in the Kepler bandpass. While most of those planets do not benefit from a known effective temperature, one has to rely on an estimate of possible equilibrium temperature domain, through an estimation of both redistribution factor $f$ and Bond albedo $A_{B}$ (see eq. 2).

$$
T_{p}=T_{\star}\left(\frac{R_{\star}}{a}\right)^{\frac{1}{2}}\left[f\left(1-A_{B}\right)\right]^{\frac{1}{4}}
$$

Comparing the range of possible equilibrium temperatures to the brightness temperature corresponding to the secondary eclipse depth allows an estimate of the upper bound for the thermal emission, and thus also an estimate of the reflected light fraction. This simple approach is however challenged by the departure from blackbody radiation of hot Jupiter thermal emission spectra. Moreover, the structure of the atmospheric temperature profile might cause visible band measurements to probe thermal emission from deep layers of the atmosphere, for instance in the absence of a stratospheric thermal inversion. Brightness temperature estimates at other wavelengths might definitely help in constraining the energy budget and sample the planetary SED. Ideally, this step would allow a constraint on the possible range of the reflected light contribution alone and exploration of the upper atmosphere properties of those irradiated planets. 

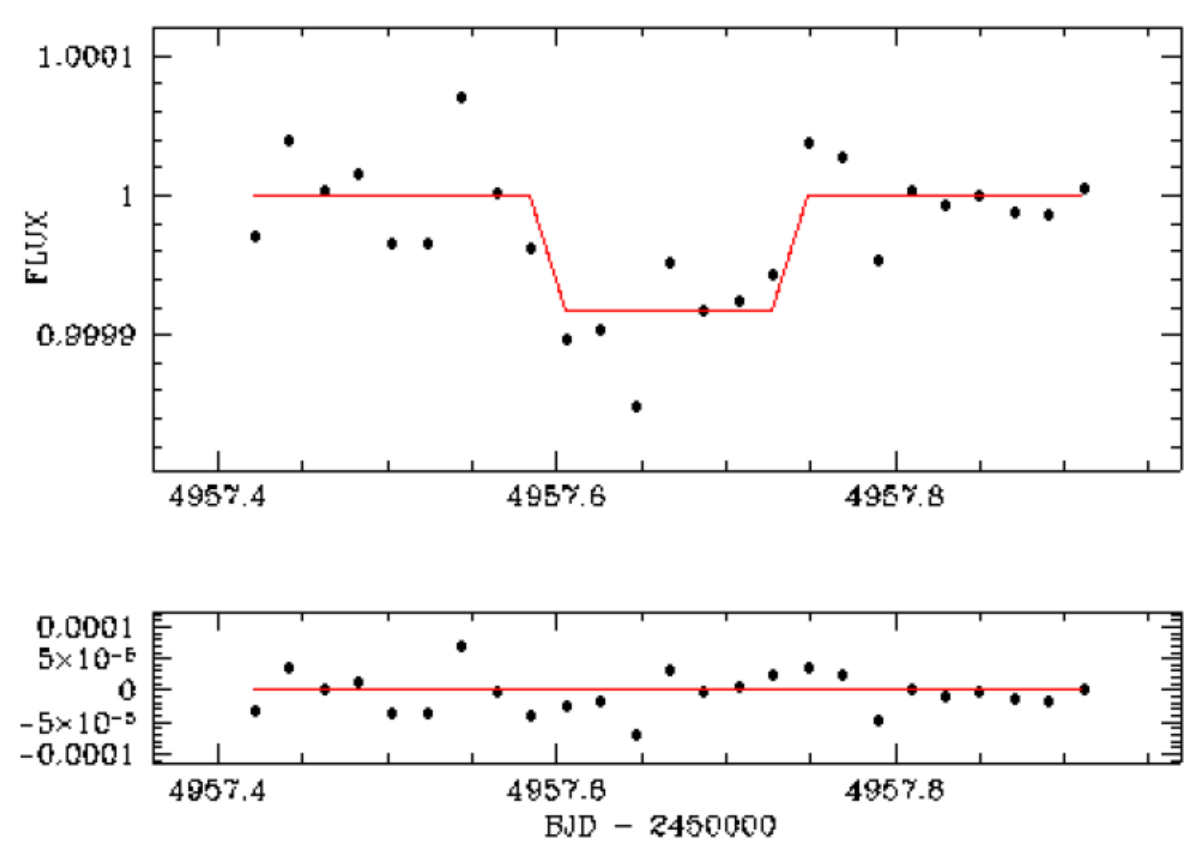

Figure 2: Top : Kepler single long-cadence (30min) secondary eclipse lightcurve of the 2.2-day period hot Jupiter HAT-P-7b with the best fit model superimposed. 1- $\sigma$ error bars are also indicated. Bottom : residuals of the fit. The occultation depth is $82 \pm$ 12 ppm. Obtained from Kepler Q1 public data, 190 ppm rms / min.

\section{Conclusions}

The Kepler mission will allow a comparative study of hot Jupiter secondary eclipses in the visible. Disentangling thermal emission from reflected light components is a critical point to address. While alkali metal absorption lines and $\mathrm{TiO}$ and $\mathrm{VO}$ molecular absorption bands are expected to shape the spectrum of hot Jupiters in the Kepler bandpass, the planetary thermal emission is arguably an important contributor for the most irradiated hot-Jupiters. Additionally, clouds are expected to form at the intersection of enstatite and iron compound condensation curves with the planetary temperature structure profile. The altitude of iron and enstatite cloud decks in hot Jupiter atmospheres significantly affects the geometric albedo. How representative are giant irradiated planets harboring high altitude reflective clouds and hazes is one of the several points Kepler will be able to address, shedding light on the properties of hot Jupiter atmospheres.

Acknowledgements. The authors want to thank the Local and Scientific Organizing Committees for a very fruitful colloquium at Haute-Provence Observatory. B.-O. D. acknowledges support from the Swiss National Science Foundation in the form of a postdoctoral fellowship. 

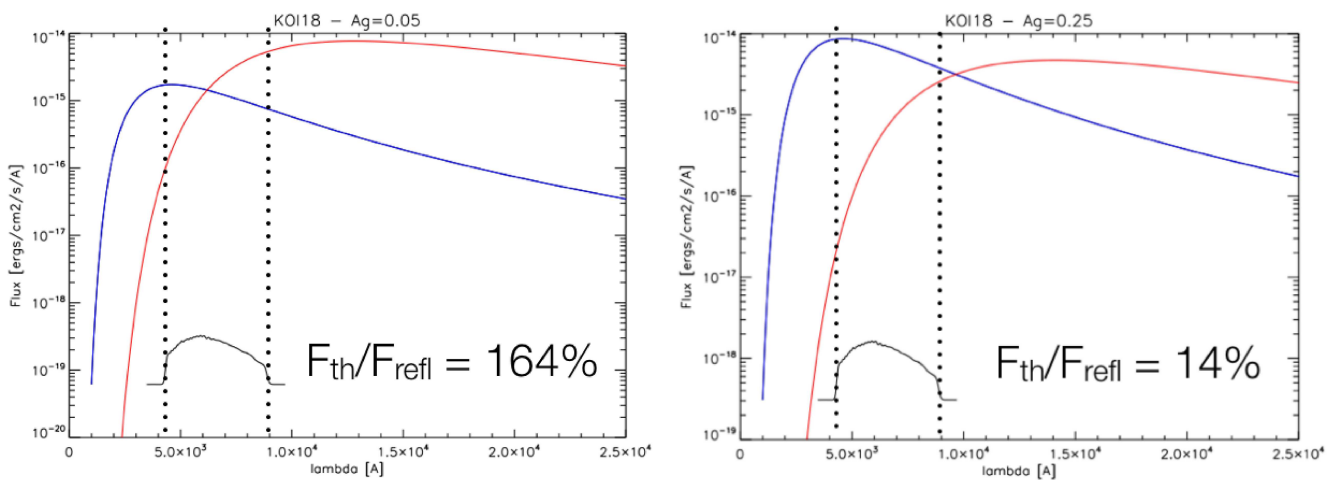

Figure 3: Thermal (red) vs. reflected (blue) components in the Kepler bandpass for a 3.5-day period and $1.4 R_{\text {Jup }}$ hot Jupiter. The low geometric albedo case (left) assumes $A_{g}=0.05$, consistent with the value determined for HD209458b by Rowe et al. 2008. The high geometric albedo case (right) explores the relative contribution of thermal and reflected fluxes for a highly irradiated and reflective hot Jupiters (e.g. Sudarsky et al. 2000) with $A_{g}=0.40$. A Lambertian sphere with no energy redistribution ( $f=2 / 3$, see Lopez-Morales $\&$ Seager 2007) are assumed (the radiative timescale is in this case shorter than the advective timescale). To estimate the planetary equilibrium temperature, the Bond albedo is computed from the geometric albedo as $A_{B}=\frac{3}{2} A_{g}$. Ratios of planetary thermal flux to reflected flux in the Kepler bandpass are indicated for each case.

\section{References}

Alonso, R., Guillot, T., Mazeh, T., Aigrain, S., Alapini, et al., 2009, in A\&A, 501, L23

Charbonneau, D., Noyes, R. W., Korzennik, S. G., Nisenson, P., Jha, S., Vogt, S. S. \& Kibrick, R. I. 1999 in ApJ, 522, L45

Jenkins, J. M., Caldwell, D. A., Chandrasekaran, H., Twicken, J. D., Bryson, S. T., et al., 2010, in ApJ, 713, L120

Kipping, D. M. \& Bakos, G. A. 2010, astro-ph.EP, 1004.3538

Leigh, C., Collier Cameron, A., Horne, K., Penny, A. \& James, D., 2003a, in MNRAS, 344, 1271

Leigh, C., Collier Cameron, A., Udry, S., Donati, J-F., Horne, K., James, D. \& Penny, A. 2003b, in MNRAS, 346, L16

López-Morales, M. \& Seager, S. 2007, in ApJ, 667, L191

Marley, M. S., Gelino, C., Stephens, D., Lunine, J. I. \& Freedman, R. 1999 in ApJ, 513, 879

Rowe, J. F. Matthews, J. M., Seager, S., Miller-Ricci, E., Sasselov, D., et al., 2008, in ApJ, 689, 1345

Seager, S. \& Deming, D. 2010, in ARA\&A, 48, 631

Seager, S. 2010, Exoplanet Atmospheres: Physical Processes (Princeton University Press)

Seager, S. \& Sasselov, D. D. 2000, in ApJ, 537, 916

Seager, S. 2003, in Earth and Planetary Science Letters, 208, 113

Snellen, I. A. G., de Mooij, E. J. W. \& Albrecht, S. 2010, in Nature, 459, 543

Sudarsky, D., Burrows, A. \& Pinto, P. 2000, in ApJ, 538, 885

Sudarsky, D., Burrows, A. \& Hubeny, I. 2003, in ApJ, 588, 1121 